Document downloaded from:

http://hdl.handle.net/10251/162603

This paper must be cited as:

Seimetz, M.; Peñas, J.; Llerena, JJ.; Benlliure, J.; García López, J.; Millán-Callado, MA.; Benlloch Baviera, JM. (2020). PADC nuclear track detector for ion spectroscopy in laserplasma acceleration. Physica Medica. 76:72-76. https://doi.org/10.1016/j.ejmp.2020.06.005

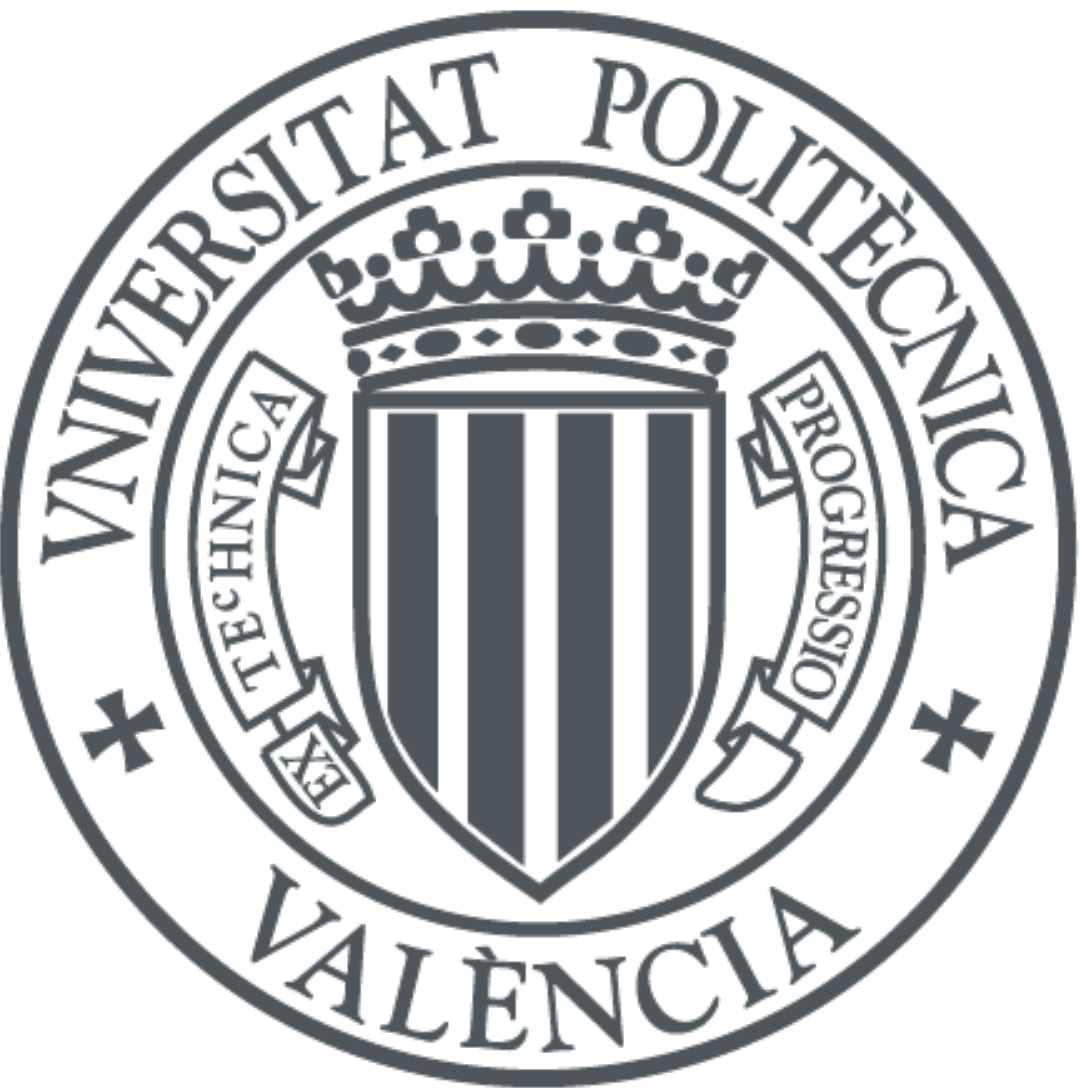

The final publication is available at

https://doi.org/10.1016/j.ejmp.2020.06.005

Copyright Elsevier

Additional Information 


\title{
PADC nuclear track detector for ion spectroscopy in laser-plasma acceleration
}

\author{
M. Seimetz ${ }^{\mathrm{a}, *}$, J. Peñas $^{\mathrm{b}}$, J.J. Llerena $^{\mathrm{b}}$, J. Benlliure $^{\mathrm{b}}$, J. García López ${ }^{\mathrm{c}, \mathrm{d}}$, \\ M.A. Millán-Callado ${ }^{c, d}$, J.M. Benlloch ${ }^{\mathrm{a}}$ \\ ${ }^{a}$ Instituto de Instrumentación para Imagen Molecular (I3M), CSIC-Universitat Politècnica \\ de València, Valencia, Spain \\ ${ }^{b}$ Instituto Galego de Física das Altas Enerxías (IGFAE), Universidade de Santiago de \\ Compostela, Santiago de Compostela, Spain \\ ${ }^{c}$ Departamento de Física Atómica, Molecular y Nuclear, Universidad de Sevilla, Sevilla, \\ Spain \\ ${ }^{d}$ Centro Nacional de Aceleradores (CNA), Universidad de Sevilla-CSIC-Junta de \\ Andalucía, Sevilla, Spain
}

\begin{abstract}
The transparent polymer polyallyl-diglycol-carbonate (PADC), also known as CR-39, is widely used as detector for heavy charged particles at low fluence. It allows for detection of single protons and ions via formation of microscopic tracks after etching in $\mathrm{NaOH}$ or $\mathrm{KOH}$ solutions. PADC combines a high sensitivity and high specificity with inertness towards electromagnetic noise. Present fields of application include laser-ion acceleration, inertial confinement fusion, radiobiological studies with cell cultures, and dosimetry of nuclear fragments in particle therapy. These require precise knowledge of the energy-dependent response of PADC to different ion species. We present calibration data for a new type of detector material, Radosys RS39, to protons (0.2-3 MeV) and carbon ions (0.6-12 MeV). RS39 is less sensitive to protons than other types of PADC. Its response to carbon ions, however, is similar to other materials. Our data indicate that RS39 allows for measuring carbon ion energies up to $10 \mathrm{MeV}$ only from the track diameters. In addition, it can be used for discrimination between protons and carbon ions in a single etching process.
\end{abstract}

Keywords: Solid state nuclear track detector; CR-39; PADC; proton; carbon

${ }^{*}$ Corresponding author

Preprint submitted to Physica Medica

May 27, 2020 
ion

\section{Background and objectives}

The transparent resin polyallyl-diglycol-carbonate (PADC, $\mathrm{C}_{12} \mathrm{H}_{18} \mathrm{O}_{7}$ ), more commonly referred to as CR-39, is a highly sensitive detector material for protons and ions. Particles with high linear energy transfer (LET) produce alterations of the molecular structure which can result in visible, microscopic tracks through a suitable etching procedure. During recent years CR-39 has become increasingly popular for applications which require the precise measurement of ion energies and particle fluence at low intensities. These comprise the clinical dose determination due to target or beam fragments in radiation therapy 1 . CR-39 has been used in cell culture studies of radiobiological effects of light ion radiation, either at classical accelerator facilities [2, 3, 4, or with protons accelerated in highly intense laser-plasma interactions [5]. In the fields of inertial confinement fusion and laser-ion acceleration CR-39 is an important diagnostic tool due to its inertness towards electrons and photons combined with high sensitivity to ions and high spatial resolution [6, 7].

The formation of visible tracks in detector materials from different providers has been consistently observed for protons between approximately $0.2 \mathrm{MeV}$ and $10 \mathrm{MeV}$ 6, 8, 9, 10, 11]. On the low-energy end, an extension of the detectable interval down to $20 \mathrm{keV}$ has been achieved [12. While the exact conditions of the etching procedure (etchant composition and concentration, temperature, etching time) have major impact on the quantitative response in terms of track diameters, the qualitative dependency on the proton energy is often similar. Specific types of CR-39 allow for detecting protons up to $18 \mathrm{MeV}$ [13] and $27 \mathrm{MeV}$ [14, respectively, due to the admixture of copolymers or antioxidants to reduce the LET threshold. As pointed out by Kodaira et al. 13], materials with high sensitivity tend to show low surface quality (and vice versa) in terms of track-like artefacts implying difficulties in automatic analysis procedures.

Various methods have been proposed to measure the energies of incident 
particles from the track characteristics in CR-39. A one-to-one correspondence between proton energies and track diameters from 0 to $1 \mathrm{MeV}$, in combination with arrangements of thin absorbers, can be exploited for the precise determination of the spectral end point in laser-ion acceleration up to a few $\mathrm{MeV}$ [11. Alternatively, differences in grey values resulting from the depth of pits can resolve ambiguities between tracks of identical diameters [15]. The energy-dependent evolution of track diameters between 0.9 and $2.5 \mathrm{MeV}$ can be utilised in a multistage etching process [16]. Protons beyond $\sim 10 \mathrm{MeV}$ penetrate through CR-39 plates of $1 \mathrm{~mm}$ thickness and may produce tracks on the rear side [17] or in subsequent layers of CR-39 stacks [18, 19. The precise, simultaneous measurement of particle energies and total fluence is necessary also for the determination of the radiation dose in experiments with biological samples.

In this work we present the first experimental data for the response of a new PADC material, Radosys RS39, to protons and carbon ions. RS39 possesses a comparatively high LET threshold corresponding to a limited energy range for the detection of protons (section 3). Under identical etching conditions track sizes are clearly different for carbon ions as compared to protons for energies above $0.2 \mathrm{MeV}$. In addition, the possibility of obtaining carbon ion energies from their track diameters is investigated (section 4).

\section{Materials and methods}

Our experiments have been carried out with two types of PADC, labelled "S" and "RS39", both provided by Radosys (Budapest, Hungary). The RS39 type, released in 2018, is manufactured with a different type of plasticiser in order to achieve better surface stability and, in this way, less amount of tracklike artefacts. The samples are of similar size $\left(10 \times 10 \mathrm{~mm}^{2}\right)$ and thickness $(0.9 \mathrm{~mm})$, with linear deviations of the order $0.05 \mathrm{~mm}$, and of the same density $\left(\rho=1.27 \mathrm{~g} / \mathrm{cm}^{3}\right)$. All samples have been etched in $6.25 \mathrm{~N} \mathrm{NaOH}$ solutions. The temperature of the etch bath (specified below for each series) has been maintained within $\pm 1^{\circ} \mathrm{C}$ of the nominal value inside a remote-controlled container. 
After etching, the front surface of the samples has been scanned with a nuclear track microscope (Radosys PT10). The photographs obtained with this system have a pixel size of $0.3 \mu \mathrm{m}$. We have used a self-made software code for the identification of circular patterns and the automatic measurement of the track diameters.

Calibration measurements with mono-energetic particle beams have been performed at the $3 \mathrm{MV}$ tandem accelerator at Centro Nacional de Aceleradores (CNA, Seville). For irradiation with protons or carbon ions under identical conditions, several samples of both types have been loaded simultaneously in a metallic holder which was placed inside a vacuum chamber at the end of the accelerator beam line. Each CR-39 plate was moved individually to the position of the beam spot which size was limited by a rhombic collimator. Proton energies $E_{p}$ from 0.7 to $3.0 \mathrm{MeV}$ have been freely adjusted; incident energies below $0.7 \mathrm{MeV}$ have been obtained by use of a $7 \mu \mathrm{m}$ thin aluminium absorber in front of the detectors. The irradiation with carbon ions in the interval $E_{C}=0.6$ 12.0 MeV has been performed without any absorbers. Different charge states have been used to quickly switch between beam energies (Table 1). When the ion enters into a solid it is immediately stripped off all electrons, so the initial charge state is expected to have only marginal influence on the track size in CR-39 [10, 17, 20]. As the particle flux with continuous beam $(\sim 10 \mathrm{pA})$ would immediately saturate the detectors a beam kicker was used to produce bunches of 25-1000 us length at $1 \mathrm{~Hz}$ pulse rate. The intensity was controlled with a scintillator-based beam monitor [21]. The total fluence on a $1 \mathrm{~cm}^{2}$ plate, estimated from the pit numbers after etching, varied between about $2 \times 10^{4}$ (where most of the tracks could be well separated) and about $1-2 \times 10^{5}$. The spatial distribution was non-uniform; even at the highest fluences areas with clear, non-overlapping tracks could be selected for image analysis. 


\begin{tabular}{|rcrc|}
\hline$E_{C}(\mathrm{MeV})$ & Charge & $E_{C}(\mathrm{MeV})$ & Charge \\
\hline 0.584 & $\mathrm{C}^{1+}$ & 4.528 & $\mathrm{C}^{2+}$ \\
0.839 & $\mathrm{C}^{2+}$ & 6.010 & $\mathrm{C}^{3+}$ \\
1.220 & $\mathrm{C}^{1+}$ & 8.057 & $\mathrm{C}^{3+}$ \\
1.701 & $\mathrm{C}^{2+}$ & 10.030 & $\mathrm{C}^{4+}$ \\
2.269 & $\mathrm{C}^{2+}$ & 12.026 & $\mathrm{C}^{5+}$ \\
3.000 & $\mathrm{C}^{3+}$ & & \\
\hline
\end{tabular}

Table 1: Carbon ion energies and respective charge states applied for sample irradiation.

\section{Calibration with proton beams}

The response of the $\mathrm{S}$ series to protons from 0 to $5.5 \mathrm{MeV}$ has been investigated in a previous publication with material produced in 2015 [11. We have repeated part of the $\mathrm{S}$ series calibration using material from 2017 in order to avoid any systematic deviations in the comparison with RS39. In the present calibration we have observed smaller tracks for $E_{p} \geq 1.5 \mathrm{MeV}$. This effect may be due to variations among different production batches of the $\mathrm{S}$ type detector material. Results are shown in Figure 1 alongside with data from literature. The latter represent the response of PADC plastics from Page Mouldings (Pershore, UK, brand name PM-355) [9] and TASL (UK, brand name TASTRAK) [6]. All data sets show a pronounced maximum in track diameters at $0.8-1 \mathrm{MeV}$; its exact position varies with the etching conditions. Etching at $70^{\circ} \mathrm{C}$ is most widely applied but the etching time necessary to remove a given bulk thickness and obtain track diameters $\geq 20 \mu \mathrm{m}$ can be reduced significantly by applying higher temperatures. For 6-6.25N NaOH baths, bulk etch rates $V_{B}=1.2-1.65 \mu \mathrm{m} / \mathrm{h}$ at $70^{\circ} \mathrm{C}$ have been reported $[9,19,22,23,24$, corresponding to a total removed thickness of 24-33 $\mu \mathrm{m}$ after 20 hours. We have measured $V_{B}$ for our etching conditions $\left(6.25 \mathrm{~N} \mathrm{NaOH}, 90^{\circ} \mathrm{C}\right)$ via 24

$$
V_{B}=\frac{1}{2} \frac{\Delta m}{\Delta t \rho A},
$$


with the sample mass lost during etching, $\Delta m$, the etching time, $\Delta t$, and the detector cross section, $A$, obtaining $V_{B}=10.9 \mu \mathrm{m} / \mathrm{h}$ for the $\mathrm{S}$ type PADC, and $V_{B}=7.6 \mu \mathrm{m} / \mathrm{h}$ for RS39. For comparison, at $98^{\circ} \mathrm{C}$ a value of $V_{B}=9.95 \mu \mathrm{m} / \mathrm{h}$ has been reported $[20$.

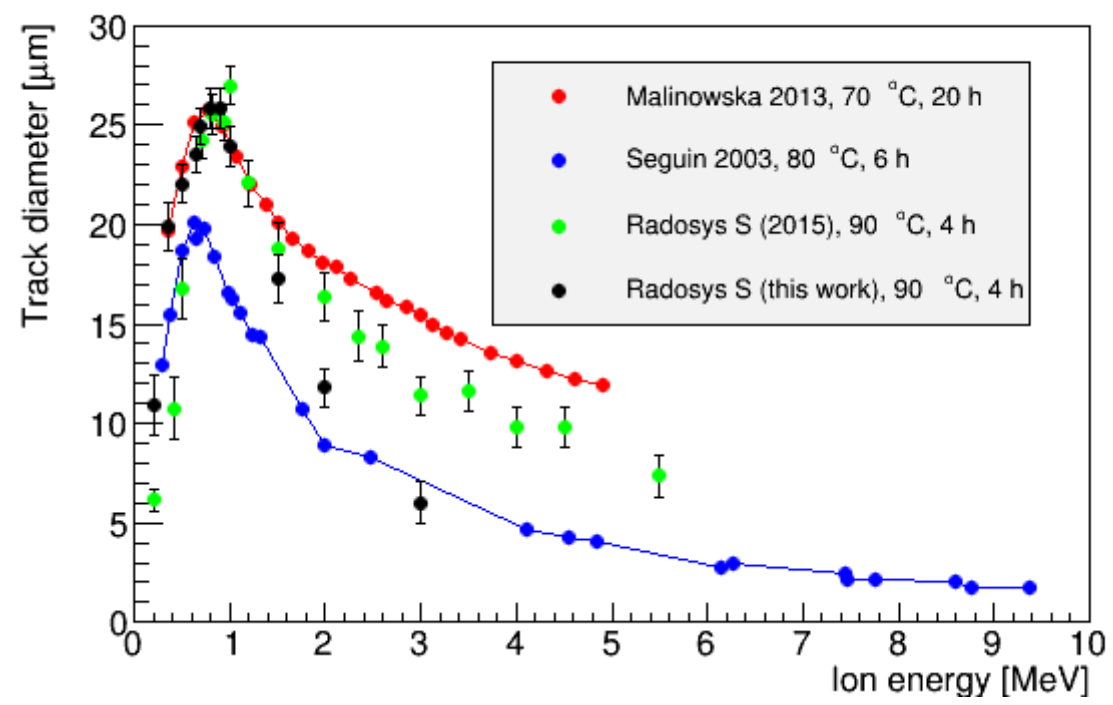

Figure 1: Track diameters of protons on $\mathrm{S}$ type PADC compared to results from literature. Lines are shown to guide the eye.

Under otherwise equal conditions proton tracks on RS39 are much smaller than on the S type PADC (Figure 2). In our experiments, microscopic pits have not been observed for $E_{p} \geq 1.5 \mathrm{MeV}$ (Figure 3). This indicates a very high LET threshold of about $20-22 \mathrm{keV} / \mu \mathrm{m}$ in water. Similarly high LET thresholds (15-22 keV/um) have been reported for TASTRAK [25]. The change in material composition from S to RS39, intended for better surface quality, correlates with reduced sensitivity, in agreement with the general observation by Kodaira et al. [13. Similar results have also been published in 1995 for a material provided by Pershore (brand name CR-39) 26, 27]. The track diameters, plottet as a function of proton energy, do not show a sharp maximum. Taking into account that the microscope resolution is typically around $1 \mu \mathrm{m}$ tracks in an energy 
interval of about $0.5 \mathrm{MeV}$ width are practically of the same size. This implies that a precise measurement of particle energies based only on the track diameters, as realised with Radosys S [11, is not possible with RS39. Nevertheless, one may use the $\mathrm{S}$ type detector to search for tracks above a certain threshold (e.g., $10 \mu \mathrm{m})$ corresponding to incident protons within a given energy range (0.4-0.9 $\mathrm{MeV}$ for $90^{\circ} \mathrm{C} / 4$ hours etching). Combinations of thin absorbers may be used to adapt more energetic protons to the detectable interval. In this way a series of intervals can be covered to determine the fluence of protons up to several $\mathrm{MeV}$.

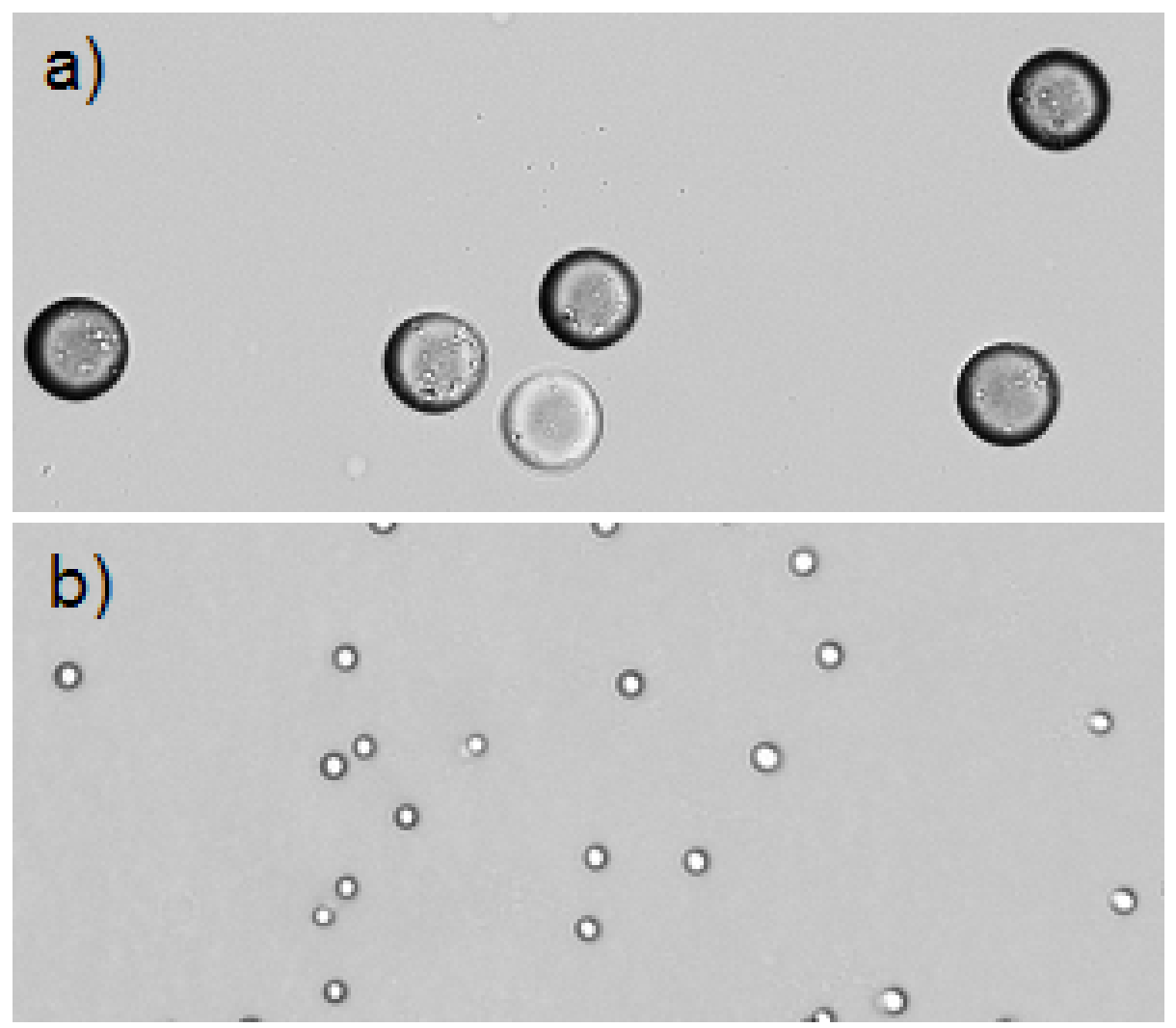

Figure 2: Microscope images of tracks from $1.0 \mathrm{MeV}$ protons on S (a) and RS39 (b). Both samples have been etched at $90^{\circ} \mathrm{C}$ during 4 hours. 


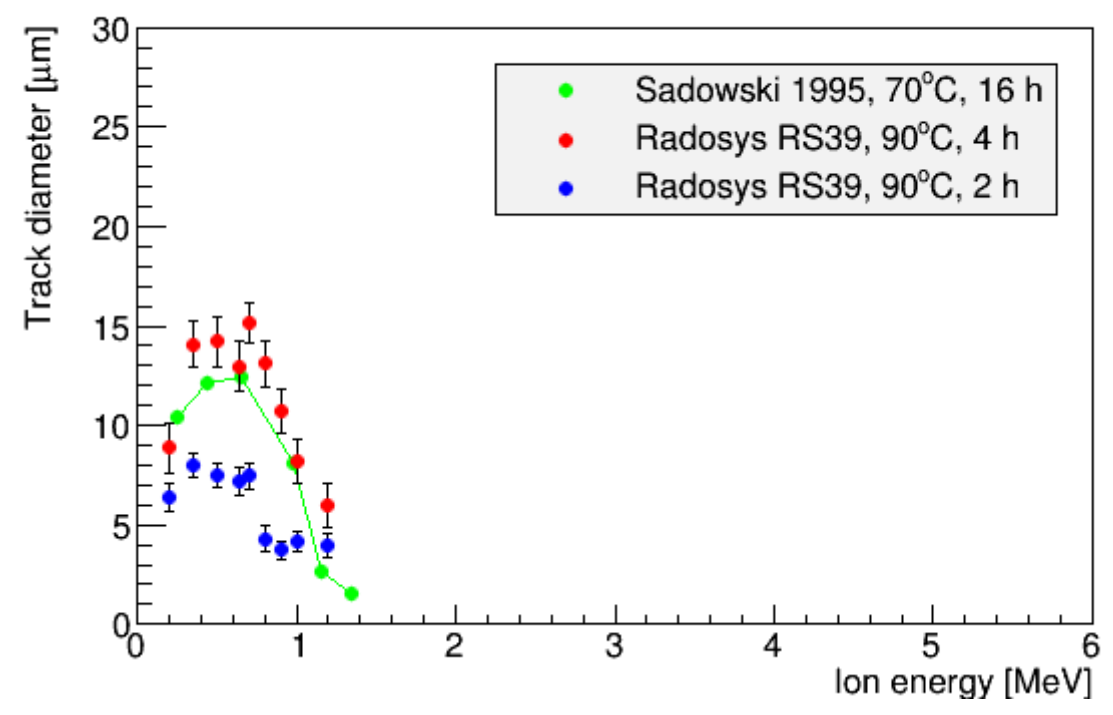

Figure 3: Track diameters of protons on RS39 type PADC compared to results from literature. Lines are shown to guide the eye.

\section{Calibration with carbon ions}

Energetic carbon ions are of increasing importance for radiation therapy. The acceleration of carbon ions by highly intense laser pulses has been demonstrated by several research groups [28, 29, 30, 31, 32, some of them using CR-39 as a detector medium [33, 34, 35, 36, 37. It is anticipated that this technique will be applied in the near future to study the radiobiological effects of carbon ions close to the Bragg peak on cell cultures. CR-39 can be used to determine the particle fluence or radiation dose in clinical research or radiation therapy [38]. By now, systematic calibrations of the energy-dependent response of PADC to carbon ions are rare; a compilation is provided in Figure 4. The data sets shown have been obtained after etching in $6-6.25 \mathrm{~N} \mathrm{NaOH}$ at $70^{\circ} \mathrm{C}$ during 6 hours unless otherwise stated. Romo and coworkers [39] presented data series starting from $1 \mathrm{MeV}$ ion energy. They did not specify the provider of their detector material and used a $6.25 \mathrm{~N} \mathrm{KOH}$ etch bath at $60^{\circ} \mathrm{C}$. Szydlowski et al. 40, tested three PADC plastics by Page Mouldings (Pershore). As the energy-dependent 
track diameters of carbon ions were similar for the brands CR-39, PM-355, and PM-500, only data points for PM-355 are included in Figure 4. More recently, data from PADC by TASL have been published for higher ion energies [17, 20. The track diameters represented by these data sets look coherent (note that only one series of each publication has been chosen). They are also consistent with our results which are the first ones obtained with Radosys RS39. The data provided by Baccou and coworkers [10, however, show much larger track diameters under the same experimental conditions and with detector material by TASL. The reason for this deviation in unknown. According to our measurements the bulk etch rate at $70^{\circ} \mathrm{C}$ is $V_{B}=1.72 \mu \mathrm{m} / \mathrm{h}$, a value close to previous publications [9, 19.

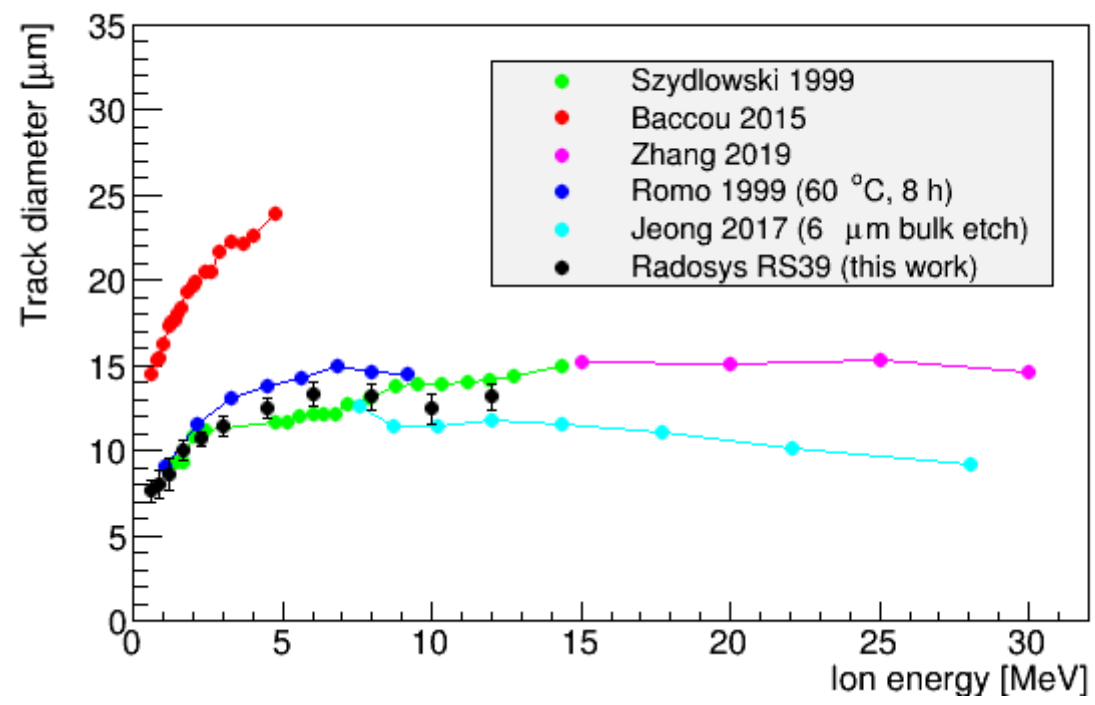

Figure 4: Track diameters of carbon ions on RS39 compared to results from literature. The etching conditions are $70^{\circ} \mathrm{C} / 6$ hours unless otherwise specified. Lines are shown to guide the eye.

At low ion energies an increase of track diameters with the particle energy has been consistently observed. This trend turns to a plateau region at about $5 \mathrm{MeV}$ under the experimental conditions of Figure 4 . With deeper etching the 
interval of strictly monotonic growth can be extended to $10 \mathrm{MeV}$ at least as shown in Figure 5 . This is also supported by data with TASL detector material [17. The absolute track sizes are larger than those of protons under the same etching conditions for energies $>0.6 \mathrm{MeV}$ (Figure 3).

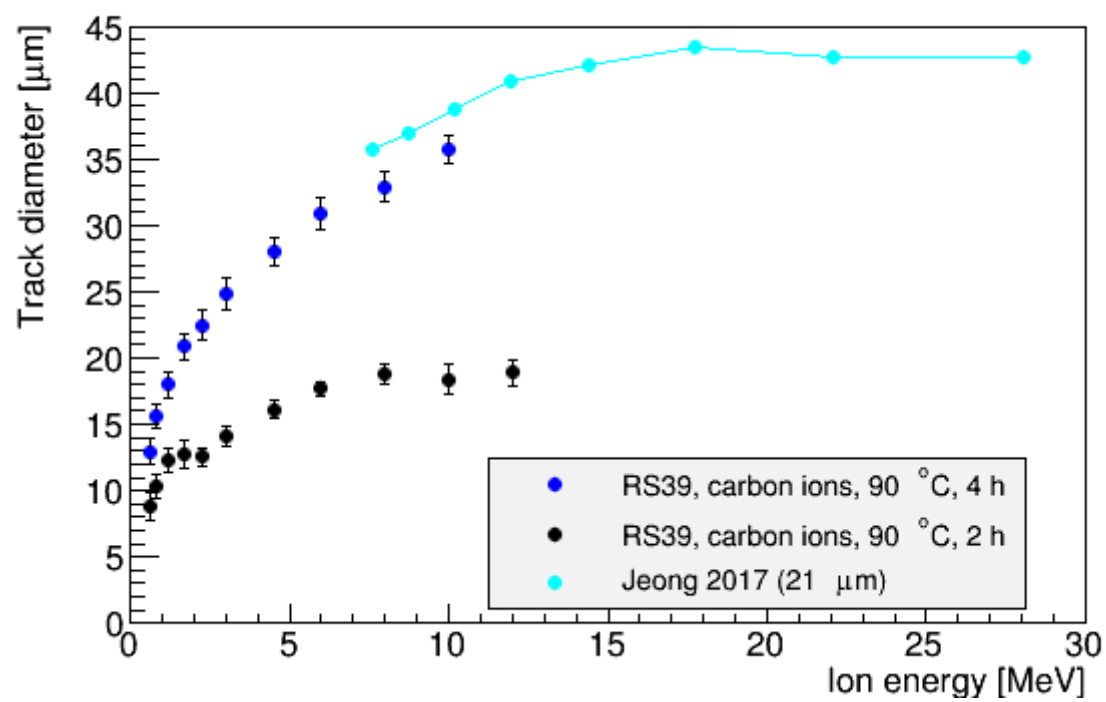

Figure 5: Track diameters of carbon ions on RS39 under the same etching conditions as those of Figure 3 One data set obtained with material by TASL is shown for comparison; lines are included to guide the eye.

\section{Conclusions}

We present experimental data of track size measurements from two PADC nuclear detector materials irradiated with mono-energetic protons and carbon ions. The calibrated interval of proton energies starts from $0.2 \mathrm{MeV}$ and covers the range of increasing track diameters and the maximum observed previously. This is of special relevance for the detection of laser-accelerated particles as these typically show broad energy distributions. The sensitivity of the RS39 type PADC to protons is limited to energies below $1.5 \mathrm{MeV}$. This material may be applied for spectroscopic measurements of protons when used in combination 
with suitable absorbers to define discrete energy intervals.

Our calibration data with carbon ions start from $0.6 \mathrm{MeV}$ and cover the interval of strictly monotonic growth which may be exploited for the measurement of ion energies directly from the track diameters. Under the same etching conditions $\left(70^{\circ} \mathrm{C}, 6\right.$ hours) our results for RS39 are in agreement with data from other nuclear track materials.

In the context of laser-ion acceleration we recommend deeper etching at $90^{\circ} \mathrm{C}$ during 4 hours as this leads to clearly visible track diameters $>10 \mu \mathrm{m}$ for protons as well as carbon ions. The two ion species can be easily distinguished since carbon ion tracks (with incident energies $>0.6 \mathrm{MeV}$ ) are larger than those of protons, making RS39 a promising material for particle discrimination. Furthermore, these conditions allow for exploiting the one-to-one correspondence of carbon ion energy and track size within a wide interval. The high temperature implies a significant reduction of the etching time; at $70^{\circ} \mathrm{C}$ about 20 hours are necessary to obtain similarly large track diameters. We have observed that still much deeper etching (e.g., at $90^{\circ} \mathrm{C} / 8$ hours) results in surface degradation and compromised track identification without offering any additional advantages.

\section{Acknowledgments}

Project funded by CSIC, grant no. 2018501082, and by the Spanish Ministerio de Ciencia, Innovación y Universidades, project MdM-2016-0692-17-2 via a predoctoral grant of type María de Maeztu FPI. Nuclear track detector material and readout equipment have been provided by Radosys Ldt. (Budapest). The authors acknowledge the contributions and commitment of the CNA accelerator operators. MS would like to thank L. Ballesteros and J. Ortiz for their support with precision equipment.

\section{References}

[1] S. Kodaira, H. Kitamura, M. Kurano, H. Kawashima, E. Benton, Contribution to dose in healthy tissue from secondary target fragments in thera- 
peutic proton, $\mathrm{He}$ and $\mathrm{C}$ beams measured with CR-39 plastic nuclear track detectors, Sci. Rep. 9 (1) (2019) 3708. doi:10.1038/s41598-019-39598-0

[2] P. Scampoli, M. Casale, M. Durante, G. Grossi, M. Pugliese, G. Gialanella, Low-energy light ion irradiation beam-line for radiobiological studies, Nuclear Instruments and Methods in Physics Research Section B: Beam Interactions with Materials and Atoms 174 (3) (2001) 337 - 343. doi:https://doi.org/10.1016/S0168-583X(00)00622-4.

[3] S. Wada, Y. Kobayashi, T. Funayama, M. Natsuhori, N. Ito, K. Yamamoto, Detection of DNA damage in individual cells induced by heavy-ion irradiation with an non-denaturing comet assay, Journal of Radiation Research 43 (Suppl) (2002) S153-S156. doi:10.1269/jrr.43.S153.

[4] S. Gaillard, D. Pusset, S. de Toledo, E. Azzam, M. Fromm, Distance distribution of bystander effects in alpha-particle irradiated cell populations using a CR-39-based culture dish, Radiation Measurements 43 (2008) S34 - S40. doi:https://doi.org/10.1016/j.radmeas.2008.03.063.

[5] A. Yogo, T. Maeda, T. Hori, H. Sakaki, K. Ogura, M. Nishiuchi, A. Sagisaka, H. Kiriyama, H. Okada, S. Kanazawa, T. Shimomura, Y. Nakai, M. Tanoue, F. Sasao, P. R. Bolton, M. Murakami, T. Nomura, S. Kawanishi, K. Kondo, Measurement of relative biological effectiveness of protons in human cancer cells using a laser-driven quasimonoenergetic proton beamline, Applied Physics Letters 98 (5) (2011) 053701. doi:10.1063/1. 3551623

[6] F. H. Séguin, J. A. Frenje, C. K. Li, D. G. Hicks, S. Kurebayashi, J. R. Rygg, B.-E. Schwartz, R. D. Petrasso, S. Roberts, J. M. Soures, D. D. Meyerhofer, T. C. Sangster, J. P. Knauer, C. Sorce, V. Y. Glebov, C. Stoeckl, T. W. Phillips, R. J. Leeper, K. Fletcher, S. Padalino, Spectrometry of charged particles from inertial-confinement-fusion plasmas, Review of Scientific Instruments 74 (2) (2003) 975-995. doi:10.1063/1.1518141. 
[7] H. Daido, M. Nishiuchi, A. S. Pirozhkov, Review of laser-driven ion sources and their applications, Reports on Progress in Physics 75 (5) (2012) 056401.

[8] N. Sinenian, M. J. Rosenberg, M. Manuel, S. C. McDuffee, D. T. Casey, A. B. Zylstra, H. G. Rinderknecht, M. G. Johnson, F. H. Séguin, J. A. Frenje, C. K. Li, R. D. Petrasso, The response of CR-39 nuclear track detector to 1-9 MeV protons, Review of Scientific Instruments 82 (2011) 103303.

[9] A. Malinowska, A. Szydłowski, M. Jaskóła, A. Korman, B. Sartowska, T. Kuehn, M. Kuk, Investigations of protons passing through the CR39/PM-355 type of solid state nuclear track detectors, Review of Scientific Instruments 84 (2013) 073511.

[10] C. Baccou, V. Yahia, S. Depierreux, C. Neuville, C. Goyon, F. Consoli, R. D. Angelis, J. E. Ducret, G. Boutoux, J. Rafelski, C. Labaune, CR-39 track detector calibration for $\mathrm{H}, \mathrm{He}$, and $\mathrm{C}$ ions from 0.1-0.5 $\mathrm{MeV}$ up to $5 \mathrm{MeV}$ for laser-induced nuclear fusion product identification, Review of Scientific Instruments 86 (2015) 083307.

[11] M. Seimetz, P. Bellido, P. García, P. Mur, A. Iborra, A. Soriano, T. Hülber, J. García López, M. C. Jiménez-Ramos, R. Lera, A. Ruiz-de la Cruz, I. Sánchez, R. Zaffino, L. Roso, J. M. Benlloch, Spectral characterization of laser-accelerated protons with CR-39 nuclear track detector, Review of Scientific Instruments 89 (2018) 023302. doi:10.1063/1.5009587.

[12] D. Xiaojiao, L. Xiaofei, T. Zhixin, H. Yongsheng, G. Shilun, Y. Dawei, W. Naiyan, Calibration of CR-39 with monoenergetic protons, Nuclear Instruments and Methods in Physics Research Section A: Accelerators, Spectrometers, Detectors and Associated Equipment 609 (2) (2009) 190 - 193. doi:https://doi.org/10.1016/j.nima.2009.08.061.

[13] S. Kodaira, K. Morishige, H. Kawashima, H. Kitamura, M. Kurano, N. Hasebe, Y. Koguchi, W. Shinozaki, K. Ogura, A performance test of 
a new high-surface-quality and high-sensitivity CR-39 plastic nuclear track detector - TechnoTrak, Nuclear Instruments and Methods in Physics Research Section B: Beam Interactions with Materials and Atoms 383 (2016) 129 - 135. doi:https://doi.org/10.1016/j.nimb.2016.07.002.

[14] K. Ogura, M. Asano, N. Yasuda, M. Yoshida, Properties of TNF-1 track etch detector, Nuclear Instruments and Methods in Physics Research Section B: Beam Interactions with Materials and Atoms 185 (2001) 222 - 227. doi:https://doi.org/10.1016/S0168-583X(01)00816-3

[15] A. Malinowska, M. Jaskóła, A. Korman, A. Szydłowski, M. Kuk, Characterization of solid state nuclear track detectors of the polyallyl-diglycolcarbonate (CR-39/PM-355) type for light charged particle spectroscopy, Review of Scientific Instruments 85 (2014) 123505. doi:10.1063/1. 4903755.

[16] F. Bahrami, F. Mianji, R. Faghihi, M. Taheri, A. Ansarinejad, Response of CR-39 to 0.9-2.5 MeV protons for $\mathrm{KOH}$ and $\mathrm{NaOH}$ etching solutions, Nuclear Instruments and Methods in Physics Research Section A: Accelerators, Spectrometers, Detectors and Associated Equipment 813 (2016) 96 -101 .

[17] T. W. Jeong, P. K. Singh, C. Scullion, H. Ahmed, P. Hadjisolomou, C. Jeon, H. Yun, K. F. Kakolee, M. Borghesi, S. Ter-Avetisyan, CR-39 track detector for multi-MeV ion spectroscopy, Scientific Reports 7 (2017) 2152.

[18] M. Kanasaki, A. Hattori, H. Sakaki, Y. Fukuda, A. Yogo, S. Jinno, M. Nishiuchi, K. Ogura, K. Kondo, K. Oda, T. Yamauchi, A high energy component of the intense laser-accelerated proton beams detected by stacked CR-39, Radiation Measurements 50 (Supplement C) (2013) 46 49.

[19] A. Groza, M. Serbanescu, B. Butoi, E. Stancu, M. Straticiuc, I. Burducea, A. Balan, A. Chirosca, B. Mihalcea, M. Ganciu, Advances in spectral dis- 
tribution assessment of laser accelerated protons using multilayer CR-39 detectors, Applied Sciences 9. doi:10.3390/app9102052.

[20] Y. Zhang, H.-W. Wang, Y.-G. Ma, L.-X. Liu, X.-G. Cao, G.-T. Fan, G.-Q. Zhang, D.-Q. Fang, Energy calibration of a CR-39 nuclear-track detector irradiated by charged particles, Nuclear Science and Techniques 30 (2019) 87. doi:10.1007/s41365-019-0619-x.

[21] M. Seimetz, P. Bellido, A. Soriano, J. G. López, M. C. Jiménez-Ramos, B. Fernández, P. Conde, E. Crespo, A. J. González, L. Hernández, A. Iborra, L. Moliner, J. P. Rigla, M. J. Rodríguez-Álvarez, F. Sánchez, S. Sánchez, L. F. Vidal, J. M. Benlloch, Calibration and performance tests of detectors for laser-accelerated protons, IEEE Transactions on Nuclear Science 62 (6) (2015) 3216-3224.

[22] M. A. Rana, I. Qureshi, Studies of CR-39 etch rates, Nuclear Instruments and Methods in Physics Research Section B: Beam Interactions with Materials and Atoms 198 (2002) 129 - 134. doi:https://doi.org/10.1016/ S0168-583X (02) 01526-4.

[23] D. Hermsdorf, M. Hunger, S. Starke, F. Weickert, Measurement of bulk etch rates for poly-allyl-diglycol carbonate (PADC) and cellulose nitrate in a broad range of concentration and temperature of $\mathrm{NaOH}$ etching solution, Radiation Measurements 42 (2007) 1-7. doi:https://doi.org/10.1016/ j.radmeas.2006.06.009.

[24] A. Azooz, M. Al-Jubbori, Interrelated temperature dependence of bulk etch rate and track length saturation time in CR-39 detector, Nuclear Instruments and Methods in Physics Research Section B: Beam Interactions with Materials and Atoms 316 (2013) 171 - 175. doi:https: //doi.org/10.1016/j.nimb.2013.09.001

[25] I. Jadrníčková, F. Spurný, To the spectrometry of linear energy transfer in charged particle beams by means of track-etch detectors, Radiation Measurements 43 (2008) S191 - S194, proceedings of the 23rd International 
Conference on Nuclear Tracks in Solids. doi:https://doi.org/10.1016/ j.radmeas.2008.04.010.

[26] M. Sadowski, E. Al-Mashhadani, A. Szydłowski, T. Czyzewski, L. Głowacka, M. Jaskóła, C. Rolfs, M. Wieluński, Comparison of responses of CR-39 and PM-355 track detectors to fast protons, deuterons and 4He ions within energy range 0.2-4.5 MeV, Radiation Measurements 25 (1995) 175 - 176. doi:https://doi.org/10.1016/1350-4487(95)00066-N

[27] M. Sadowski, A. Szydłowski, M. Jaskóła, T. Czyzewski, A. Kobzev, Comparison of responses of CR-39, PM-355, and CN track detectors to energetic hydrogen-, helium-, nitrogen-, and oxygen-ions, Radiation Measurements 28 (1997) 207 - 210. doi:https://doi.org/10.1016/S1350-4487(97) 00069-3.

[28] A. Henig, S. Steinke, M. Schnürer, T. Sokollik, R. Hörlein, D. Kiefer, D. Jung, J. Schreiber, B. M. Hegelich, X. Q. Yan, J. Meyer-ter Vehn, T. Tajima, P. V. Nickles, W. Sandner, D. Habs, Radiation-pressure acceleration of ion beams driven by circularly polarized laser pulses, Phys. Rev. Lett. 103 (2009) 245003. doi:10.1103/PhysRevLett.103.245003.

[29] S. Kar, K. F. Kakolee, B. Qiao, A. Macchi, M. Cerchez, D. Doria, M. Geissler, P. McKenna, D. Neely, J. Osterholz, R. Prasad, K. Quinn, B. Ramakrishna, G. Sarri, O. Willi, X. Y. Yuan, M. Zepf, M. Borghesi, Ion acceleration in multispecies targets driven by intense laser radiation pressure, Phys. Rev. Lett. 109 (2012) 185006. doi:10.1103/PhysRevLett. 109.185006.

[30] S. Palaniyappan, C. Huang, D. C. Gautier, C. E. Hamilton, M. A. Santiago, C. Kreuzer, A. B. Sefkow, R. C. Shah, J. C. Fernández, Efficient quasi-monoenergetic ion beams from laser-driven relativistic plasmas, Nature Communications 6 (2015) 10170. doi:https://doi.org/10.1038/ ncomms 10170 
[31] C. McGuffey, A. Raymond, T. Batson, R. Hua, G. M. Petrov, J. Kim, C. M. Krauland, A. Maksimchuk, A. G. R. Thomas, V. Yanovsky, K. Krushelnick, F. N. Beg, Acceleration of high charge-state target ions in highintensity laser interactions with sub-micron targets, New Journal of Physics 18 (2016) 113032. doi:10.1088/1367-2630/18/11/113032.

[32] W. J. Ma, I. J. Kim, J. Q. Yu, I. W. Choi, P. K. Singh, H. W. Lee, J. H. Sung, S. K. Lee, C. Lin, Q. Liao, J. G. Zhu, H. Y. Lu, B. Liu, H. Y. Wang, R. F. Xu, X. T. He, J. E. Chen, M. Zepf, J. Schreiber, X. Q. Yan, C. H. Nam, Laser acceleration of highly energetic carbon ions using a double-layer target composed of slightly underdense plasma and ultrathin foil, Phys. Rev. Lett. 122 (2019) 014803. doi:10.1103/PhysRevLett.122.014803.

[33] M. Hegelich, S. Karsch, G. Pretzler, D. Habs, K. Witte, W. Guenther, M. Allen, A. Blazevic, J. Fuchs, J. C. Gauthier, M. Geissel, P. Audebert, T. Cowan, M. Roth, MeV ion jets from short-pulse-laser interaction with thin foils, Phys. Rev. Lett. 89 (2002) 085002. doi:10.1103/PhysRevLett. 89.085002 .

[34] A. Henig, D. Kiefer, K. Markey, D. C. Gautier, K. A. Flippo, S. Letzring, R. P. Johnson, T. Shimada, L. Yin, B. J. Albright, K. J. Bowers, J. C. Fernández, S. G. Rykovanov, H.-C. Wu, M. Zepf, D. Jung, V. K. Liechtenstein, J. Schreiber, D. Habs, B. M. Hegelich, Enhanced laser-driven ion acceleration in the relativistic transparency regime, Phys. Rev. Lett. 103 (2009) 045002. doi:10.1103/PhysRevLett.103.045002.

[35] D. C. Carroll, O. Tresca, R. Prasad, L. Romagnani, P. S. Foster, P. Gallegos, S. Ter-Avetisyan, J. S. Green, M. J. V. Streeter, N. Dover, C. A. J. Palmer, C. M. Brenner, F. H. Cameron, K. E. Quinn, J. Schreiber, A. P. L. Robinson, T. Baeva, M. N. Quinn, X. H. Yuan, Z. Najmudin, M. Zepf, D. Neely, M. Borghesi, P. McKenna, Carbon ion acceleration from thin foil targets irradiated by ultrahigh-contrast, ultraintense laser pulses, New 
Journal of Physics 12 (2010) 045020. doi:10.1088/1367-2630/12/4/ 045020 .

[36] D. Jung, L. Yin, B. J. Albright, D. C. Gautier, S. Letzring, B. Dromey, M. Yeung, R. Hörlein, R. Shah, S. Palaniyappan, K. Allinger, J. Schreiber, K. J. Bowers, H.-C. Wu, J. C. Fernández, D. Habs, B. M. Hegelich, Efficient carbon ion beam generation from laser-driven volume acceleration, New Journal of Physics 15 (2013) 023007. doi:10.1088/1367-2630/15/ 2/023007.

[37] F. Dollar, C. Zulick, T. Matsuoka, C. McGuffey, S. S. Bulanov, V. Chvykov, J. Davis, G. Kalinchenko, G. M. Petrov, L. Willingale, V. Yanovsky, A. Maksimchuk, A. G. R. Thomas, K. Krushelnick, High contrast ion acceleration at intensities exceeding $10^{21} \mathrm{Wcm}^{-2}$, Physics of Plasmas 20 (2013) 056703. doi:10.1063/1.4803082.

[38] R. Kohno, N. Yasuda, H. Takeshi, Y. Kase, K. Ochiai, M. Komori, N. Matsufuji, T. Kanai, Measurements of dose-averaged linear energy transfer distributions in water using CR-39 plastic nuclear track detector for therapeutic carbon ion beams, Japanese Journal of Applied Physics 44 (2005) 8722-8726. doi:10.1143/jjap.44.8722.

[39] V. Romo, J. Rickards, G. Espinosa, J. Golzarri, The Response of CR-39 Polycarbonate to Energetic Carbon Ions, Radiation Protection Dosimetry 85 (1999) 459-461. doi:10.1093/oxfordjournals.rpd.a032897.

[40] A. Szydłowski, T. Czyzewski, M. Jaskóła, M. Sadowski, A. Korman, J. Kedzierski, W. Kretschmer, Investigation of response of CR-39, PM355 and PM-500 types of nuclear track detectors to energetic carbon ions, Radiation Measurements 31 (1999) 257 - 260. doi:https://doi.org/10. 1016/S1350-4487(99)00125-0. 